\title{
How does the kinase Lck phosphorylate the T cell receptor? Spatial organization as a regulatory mechanism
}

\section{Jérémie Rossy, David J. Williamson and Katharina Gaus*}

Centre for Vascular Research, University of New South Wales, Sydney, NSW, Australia

\section{Edited by:}

Michael Dustin, Skirball Institute of

Biomolecular Medicine, New York

University School of Medicine, USA

Reviewed by:

Michael Dustin, Skirball Institute of

Biomolecular Medicine, New York

University School of Medicine, USA

Christopher E. Rudd, University of

Cambridge, UK

\section{${ }^{*}$ Correspondence:}

Katharina Gaus, Centre for Vascular Research, University of New South Wales, Sydney, NSW 2052, Australia.

e-mail:k.gaus@unsw.edu.au
T cell signaling begins with the ligation of the T cell antigen receptor (TCR) by a cognate peptide and the phosphorylation of the receptor's immunoreceptor tyrosine-based activation motif domains by the kinase Lck. However, the canonical receptor model is insufficient to explain how the constitutively active kinase Lck can discriminate between non-ligated and ligated TCRs. Here, we discuss the factors that are thought to regulate the spatial distribution of the TCR and Lck, and therefore critically influence TCR signaling initiation.

Keywords: membrane organization, $\mathrm{T}$ cell receptor, Lck kinase, signal transduction, protein distribution

\section{INTRODUCTION}

The family of Src kinases play key roles in the signal transduction of many cell surface receptors in a diverse range of cellular functions, such as cell growth, differentiation, migration, and survival (Bromann et al., 2004; Parsons and Parsons, 2004). In $\mathrm{T}$ cells, the lymphocyte-specific protein tyrosine kinase (Lck) is critical in the early propagation and modulation of $\mathrm{T}$ cell receptor (TCR) signaling. TCR signaling is triggered by the recognition and engagement of antigenic peptides bound to major histocompatibility complex (MHC) and relies on the phosphorylation by Lck of receptor complex at immunoreceptor tyrosine-based activation motif (ITAM) consensus sites. Phosphorylated ITAMs recruit zeta-chain-associated protein kinase 70 (Zap70), which requires additional phosphorylation by Lck to be activated (Wange and Samelson, 1996; Palacios and Weiss, 2004). Zap70 in turn phosphorylates other proteins in TCR signaling cascade, which eventually leads to $\mathrm{T}$ cell activation.

Lck is bound to the plasma membrane via myristoylated and palmitoylated residues at its $\mathrm{N}$-terminus. It also contains a SH3 and a SH2 domains next to the membrane anchor followed by a catalytic tyrosine kinase domain and a short C-terminal tail. As an essential kinase in the propagation of TCR signaling, the regulation of Lck activity is itself tightly controlled by conformational changes mainly relying on phosphorylation and dephosphorylation on two regulatory tyrosine residues. When the C-terminal inhibitory tyrosine (Tyr505) is phosphorylated, it can interact with its own $\mathrm{SH} 2$ domain, which causes an intra-molecular arrangement and locks the kinase in an inactive or "closed" conformation (Figure 1). SH3 and SH2 domains intra molecular interactions also contribute to stabilize the closed conformation (Boggon and Eck, 2004). In contrast, phosphorylation of the activating tyrosine (Tyr394) stabilizes the activation loop in an active conformation (Yamaguchi and Hendrickson, 1996; Boggon and Eck, 2004). An intermediary form in which Lck is phosphorylated on both regulatory tyrosine residues also exists and is catalytically active. This double phosphorylated form accounts for $\sim 20 \%$ of all Lck in T lymphocyte (D'Oro and Ashwell, 1999; Nika et al., 2010). The inhibitory Tyr505 residue is phosphorylated by C-terminal Src kinase (Csk) and dephosphorylated by the tyrosine phosphatase CD45 (Alexander, 2000; Palacios and Weiss, 2004). The activating Tyr394 residue is phosphorylated by Lck in a process of transphosphorylation. Tyr394 is also dephosphorylated by SH2 domain-containing phosphatase-1 (SHP-1; Stefanová et al., 2003) and CD45, which can hence positively and negatively regulate Lck activity (Alexander, 2000; Saunders and Johnson, 2010). Currently the consensus is that $\mathrm{CD} 45$ acts predominately negatively on Lck activity (D'Oro and Ashwell, 1999; Wong et al., 2008) because high levels of CD45 are required to dephosphorylate Y394 while low levels are sufficient for Y505 dephosphorylation and Lck activation (McNeill et al., 2007; Zikherman et al., 2010). This suggests that Lck is kept inactive by high level of CD45 in resting cells, whereas partial segregation of the two molecules upon TCR activation reduces the levels of $\mathrm{CD} 45$ and favors dephosphorylation at Y505 and hence Lck activation. All in all, the various combinations of kinases and phosphatase accounts for the complex regulatory mechanism of Lck (Figure 1).

In resting T lymphocytes, a large percentage of Lck is constitutively active. More surprisingly, however, TCR activation has no impact on Lck activity with the proportion of the different phosphorylation and conformational states of Lck remaining relatively unchanged (Secrist et al., 1993; Paster et al., 2009; Nika et al., 2010). This implies that a pool of constitutively active Lck is responsible for the propagation of TCR signaling. Lck activity is thought to be held in check under resting conditions by phosphatases and Csk. Consequently, suppression of phosphatases activity by pervanadate treatment markedly enhances phosphorylation of Y394 and promotes T cell activation (Secrist et al., 1993). Similarly, inhibiting Csk activity triggers phosphorylation of Lck 

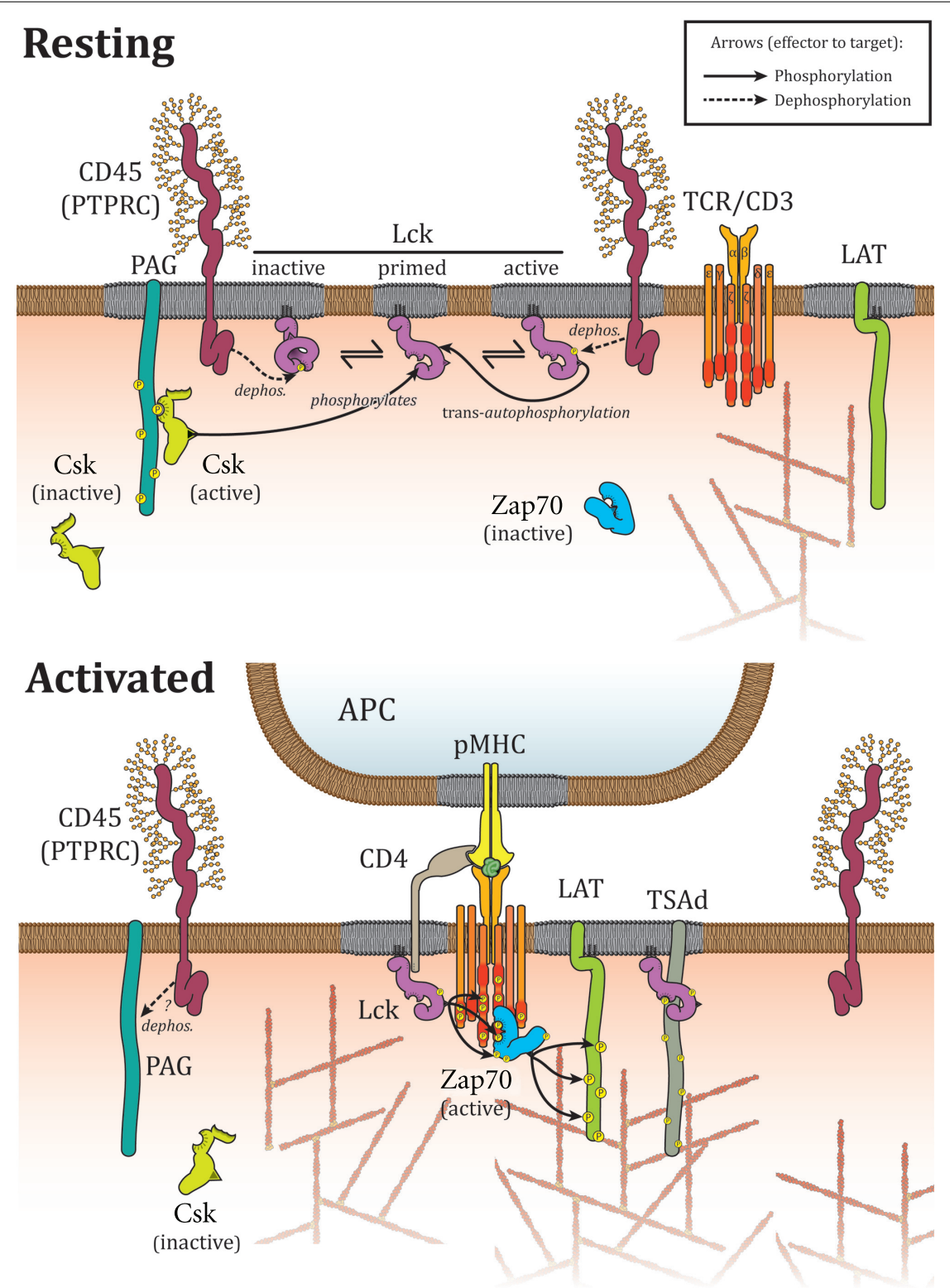

FIGURE 1 | Interactions controlling Lck localization upon TCR activation. In resting cells, Lck is located in microdomains where it interacts directly with CD45 that acts both on activating Tyr394 and inhibiting Tyr505. Csk is targeted to membrane microdomains by PAG and aids to limit Lck activity via Tyr505 phosphorylation. Association with membrane domains and protein-protein interactions prevent constitutively active Lck to phosphorylate TCR or Zap70 in resting cells. Upon activation, CD45 is excluded from membrane microdomains and Csk detaches from the plasma membrane as a consequence of PAG dephosphorylation by CD45. Lck oligomerizes, binds to and phosphorylates TCR and Zap70 to mediate TCR signaling Interactions with TSAd and CD4/8 contribute to enhance TCR binding and signaling. at Tyr394 and TCR signaling (Schoenborn et al., 2011). Taken together, it seems that Lck kinase activity and TCR phosphorylation are critically regulated by the interaction probabilities of kinases, phosphatases, and their substrates.

\section{SPATIAL PATTERNING OF THE IMMUNOLOGICAL SYNAPSE}

Since the binding of TCR to antigenic peptides does not increase the proportion of active Lck, the mechanisms that allow the kinase to initiate signaling only when the receptor is peptide-bound are likely to depend on interaction probabilities and hence spatial distributions within the plasma membrane of Lck relative to its modulators and targets. In this model, ITAM phosphorylation is balanced by the access of constitutively active Lck to TCRs relative to the action of phosphatases. To characterize the spatial patterns of signaling proteins at $\mathrm{T}$ cell activation sites and immunological synapses and identify the mechanisms of the lateral membrane organization has therefore been a long-standing goal of many researchers.

When stimulated with antigen presenting cells, TCR and signaling proteins redistribute to first form so-called TCR microclusters 
(Seminario and Bunnell, 2008) and then the bull-eye pattern of a mature immunological synapse (Grakoui et al., 1999; Dustin, 2009). The diffusion of Lck molecules within the plasma membrane is indeed affected by TCR activation: Lck molecules display heterogeneous diffusion in activated cells with an increased residency time in microdomains that contain TCR (Ike et al., 2003; Douglass and Vale, 2005). Lck distribution matches that of TCR in activated cells, and both molecules are recruited simultaneously to the immunological synapse upon activation (Ehrlich et al., 2002) where they co-localize in the center of the synapse (Monks et al., 1998; Freiberg et al., 2002). However, active Lck is found at the periphery of the immunological synapse, where it initiates TCR phosphorylation and signaling cascades (Campi et al., 2005). Interestingly, TCR clusters form before and independently of Lck (Campi etal., 2005) suggesting that Lck is somehow recruited to pre-formed TCR clusters. Importantly, CD45 and Lck have distinct and mutually exclusive localizations in activated T cells, consistent with the notion that upon TCR triggering, CD45 mainly limits Lck activity and TCR phosphorylation (D'Oro and Ashwell, 1999; McNeill et al., 2007; Wong et al., 2008). Several studies report CD45 exclusion from TCRcontaining areas within the immunological synapse (Johnson, 2000; Leupin et al., 2000) and more precisely from TCR microclusters (Varma et al., 2006; Kaizuka et al., 2009). Additionally, CD45 is excluded from CD2 clusters that contain Lck and the adaptor linker for activation of T cells (LAT; Douglass and Vale, 2005). Similarly, Csk loses its membrane association upon T cell activation, segregating de facto from membrane-associated Lck (Brdika et al., 2000; Davidson et al., 2003). In summary, the distribution of Lck is modified by TCR engagement, resulting in the recruitment of Lck to TCR microclusters and segregation of Lck from CD45 and Csk. However, the molecular mechanisms of these redistributions are poorly understood. Below, we discuss the interactions that may regulate Lck localization and distribution.

\section{INTERACTIONS GOVERNING LcK LOCALIZATION AND DISTRIBUTION}

The first described mechanism that controls Lck localization is the binding of Lck to the coreceptors CD4 and CD8. Lck directly associate with CD4 and CD8 in T cells (Rudd et al., 1988; Veillette et al., 1988) - an interaction that is mediated by zinc, which allows the cytoplasmic tails of CD4/8 to interact with the N-terminal region of Lck and to form heterodimers (Lin et al., 1998; Kim et al., 2003). The major function of CD4/8 is to enhance the recruitment of Lck to the immunological synapse (Holdorf et al., 2002) and deliver Lck to the TCR-pMHC complex (Li et al., 2004; Van Laethem et al., 2007; Artyomov et al., 2010). However, the TCR complex was able to induce signaling even the absence of coreceptors (Locksley et al., 1993; Schilham et al., 1993; Van Laethem et al., 2007), emphasizing a role for CD4/8 mediated-delivery of Lck in enhancing specificity of TCR binding to pMHC rather than being a prerequisite for TCR signaling.

Protein-protein interactions play a dominant role in membrane distributions since such interactions regulate diffusion and often result in immobile clusters. The $\mathrm{SH} 2$ domain of Lck, which is essential for TCR signaling, is a mediator of such protein interactions (Table 1). In the inactive conformation, the $\mathrm{SH} 2$ domain of Lck binds to the inhibitory phosphotyrosine (pTyr505) of Lck, preventing interactions with other proteins (Eck et al., 1994; $\mathrm{Xu}$ et al., 1999). However, the affinity of the SH2 domain for pTyr505 is relatively weak (Nika et al., 2007) allowing competitive binding with other proteins such as TCR and Zap70 (Duplay et al., 1994; Straus et al., 1996; Yamasaki et al., 1996). Interestingly, it is the phosphotyrosine binding property of the $\mathrm{SH} 2$ domain that mediates the interactions with the phosphorylated $\zeta$ chain of TCR and phosphorylated Zap70 (Lewis et al., 1997). Thus, the SH2 domain can maintain a stable association of Lck with its main targets after they have been phosphorylated, effectively consolidating the protein network for sustained signaling and signal amplification (Pawson, 2004). After TCR engagement, oligomerization of Lck, driven by a "head-to-toe" SH2 and SH3 domains association (Lee-Fruman etal., 1996) likely contributes to the amplification of TCR signaling. However, to which extend specific SH2 interactions are essential or could substitute CD4/8 coreceptor engagement is currently not clear. The SH2 domains may also confer intrinsic adaptor properties to Lck, as a potential kinase independent function (Xu and Littman, 1993).

$\mathrm{SH} 2$ domain interactions not only regulate Lck access to TCR and Zap70, but also take part in the control of Lck

\section{Table 1 | Lck molecular interactions.}

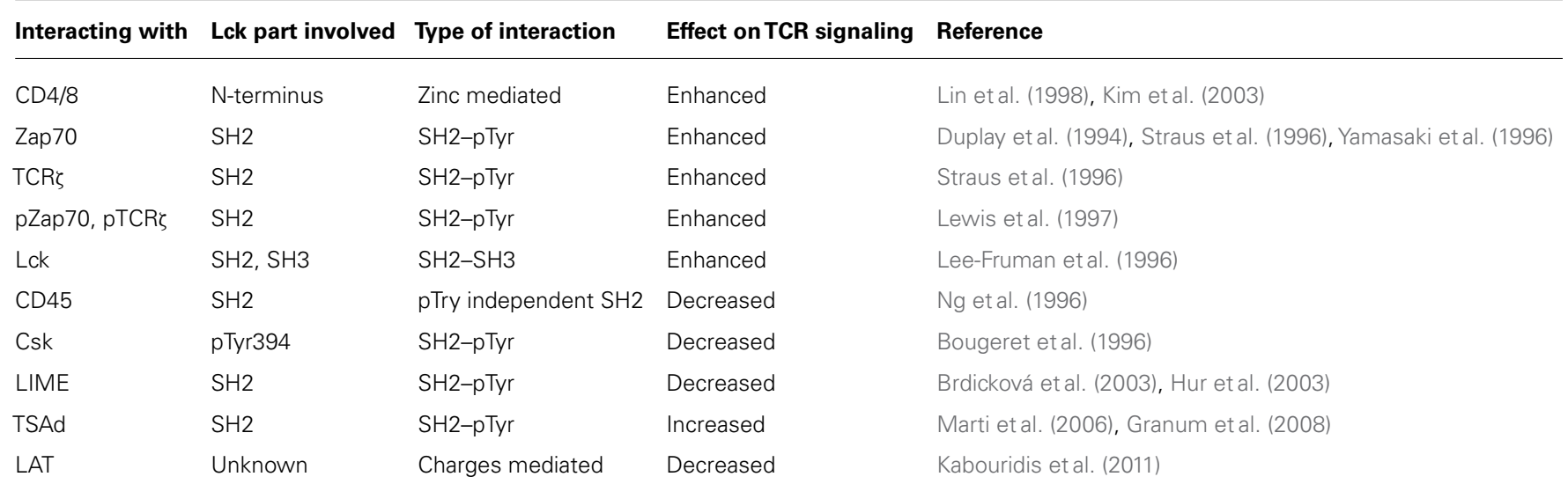


distribution relative to proteins that regulate its activity (Table 1). A phosphotyrosine-independent interaction mediates the binding of Lck's SH2 domain to CD45 (Ng et al., 1996). Additionally, the SH2 domain of Csk binds to activating pTyr394 in Lck (Bougeret etal., 1996), probably preventing Lck hyperactivation. Lck and Csk interact indirectly as well, via SH2 mediated binding to the adaptor LIME (Brdicková et al., 2003; Hur et al., 2003). The SH2 domains of Lck also bind to the T cell-specific adapter protein (TSAd) that in turn positively regulates Lck activity upon TCR activation (Marti et al., 2006; Granum et al., 2008). Hence, the SH2 domain of Lck does not only contribute to the enhancement and propagation of TCR signaling, but also participate in regulating Lck activity by linking it to CD45 Csk and TSAd. Additionally, direct interaction of $\mathrm{CD} 45$ with $\mathrm{CD} 3 \zeta$ ITAM domains may contribute to the differential localization and interaction of Lck and CD45 in resting and activated cells (Furukawa, 1994).

Other SH2 domain-independent protein-protein interactions also play a role in controlling Lck distribution. The diffusion and localization of membrane proteins including Lck can also be regulated by the actin cytoskeleton. Actin polymerization is essential in the formation of TCR microclusters, but is not required for their maintenance once they are established (Campi et al., 2005; Seminario and Bunnell, 2008; Kaizuka et al., 2009). Lck associates with actin upon TCR engagement, and is responsible for the SH2 domain-dependant anchoring of TCRS to the actin cytoskeleton (Rozdzial et al., 1998). However, more evidence is required to understand how Lck distribution is regulated by the actin cytoskeleton. Other protein-protein interactions of Lck with effectors include binding to LAT, which is recruited and phosphorylated by Zap70 and interacts with the active form of Lck upon TCR stimulation. Lck interaction with LAT is electrostatic and impacts negatively on Lck activity (Kabouridis et al., 2011). Finally, extracellular proteinprotein interactions can also act on Lck distribution: a lattice formed by galectin, a glycoprotein binding protein, can contribute to maintain Lck and CD45 in the common membrane microdomains in resting cells, favoring Lck inhibition by CD45 (Chen et al., 2007).

It has previously suggested that residency in membrane microdomains or lipid rafts controls Lck distribution relative to effectors and targets. The myristoylation and palmitoylation of Lck's membrane binding domain target the kinase to cholesterol- and glycolipid-enriched microdomains (ShenoyScaria et al., 1994; Filipp et al., 2003; Rodgers et al., 2005). These membrane microdomains are thought to aid the translocation of Lck to the immunological synapse after activation by antigen presenting cells (Jordan and Rodgers, 2003). More specifically, active Lck translocates to lipid rafts upon TCR engagement (Filipp et al., 2003). Such membrane compartmentalization is especially important for the regulation of Lck distributions relative to CD45 and Csk, thereby governing Lck activation status (Figure 1). Indeed, replacing the membrane targeting motif of Lck with that of Fyn results in aberrant TCR signaling (Salmond et al., 2011) and hyperphosphorylation of the inhibitory Tyr505 (Gervais and Veillette, 1995). However, even though TCR activation reduces CD45 association with lipid rafts (Edmonds and Ostergaard, 2002), the evidence whether CD45 and Lck localization in the same microdomains favors or prevents Lck activation is confusing (Rodgers and Rose, 1996; Chichili et al., 2010). PAG/Cbp is a raft-associated membrane protein that interacts with Csk and localizes the kinase to lipid rafts in resting cells (Brdika etal., 2000; Kawabuchi et al., 2000) where it contributes to Lck inhibition by phosphorylating the inhibitory Tyr505. Upon T cell activation, PAG is dephosphorylated and Csk is removed from the plasma membrane to allow Lck activation (Brdika et al., 2000; Davidson et al., 2003). Other evidence for the importance of lipid domains comes from coreceptors: CD28 induces the recruitment of Lck to lipid rafts (Tavano et al., 2006), where Lck associates with CD4, which in turn controls raft aggregation (Fragoso et al., 2003). However, the controversy about methodologies used lipid raft studies (Munro, 2003) and the questions about their very existence (Nichols, 2005) as well as the fact that CD45 can either activate and deactivate Lck (Saunders and Johnson, 2010) prevent a clear understanding of the lipid rafts role in the control of Lck distribution. In a landmark study, Douglass and Vale (2005) have shown that the membrane anchor of Lck that targets the proteins to lipid raft does not control Lck diffusion and microclusters localization. Although membrane lipid composition is likely to be involved in the control of Lck distribution, it could be via other mechanisms, such as the electrostatic effects of the charges-mediated binding of TCR $\zeta$ chain basic residues to the plasma membrane, which controls TCR-Lck interactions and hence localizations (Zhang et al., 2011). It should also be noted that spatial control of Lck distribution can be achieved without proteins or lipids interactions as proposed by the kinetic-segregation model. This model postulates that close-contacts zones are created when engaged TCR shorten the distance between $\mathrm{T}$ cell and APC membranes. CD45 that bears a large ectodomain is excluded from these areas, generating a favorable environment for TCR and Zap70 phosphorylation by Lck (Davis and van der Merwe, 2006).

\section{CONCLUSION}

Since Lck is constitutively active and TCR engagement does not modulate Lck activity per se, it is thought that the spatial distributions of Lck relative to its substrates and other kinases and phosphatases minimize ITAM phosphorylation in resting $\mathrm{T}$ cells while fast and efficiently phosphorylating the receptor in activated cells. Evidence has been presented for various mechanisms that may regulate Lck localizations in the plasma membrane such as SH2 domain-mediated interactions with TCR signaling proteins and Lck activity-regulating proteins, $\mathrm{SH} 2$ domain-independent scaffolding functions, association with lipid rafts and redistribution caused by the close contact zone between cellular membranes. It is very likely that Lck localization is regulated by the same processes that allow the formation of compositionally distinct TCR microclusters and synapse patterns upon TCR triggering. New single-molecule fluorescence microscopy methods that measure protein distributions with nanometer precision may afford new insights into protein distributions during $\mathrm{T}$ cell activation (Lillemeier et al., 2010; Williamson et al., 2011) that allow us to determine the interdependencies between Lck activity, conformational states and TCR phosphorylation. 


\section{REFERENCES}

Alexander, D. R. (2000). The CD45 tyrosine phosphatase: a positive and negative regulator of immune cell function. Semin. Immunol. 12, 349-359.

Artyomov, M. N., Lis, M., Devadas, S., Davis, M. M., and Chakraborty, A. K. (2010). CD4 and CD8 binding to MHC molecules primarily acts to enhance Lck delivery. Proc. Natl. Acad. Sci. U.S.A. 107, 16916-16921.

Boggon, T. J., and Eck, M. J. (2004). Structure and regulation of Src family kinases. Oncogene 23, 7918-7927.

Bougeret, C., Delaunay, T., Romero, F., Jullien, P., and Siegmund, F. (1996). Detection of a physical and functional interaction between Csk and Lck which involves the $\mathrm{SH} 2$ domain of Csk and is mediated by autophosphorylation of Lck on tyrosine 394. J. Biol. Chem. 271, 7465-7472.

Brdicková, N., Brdicka, T., Angelisová, P., Horváth, O., Spicka, J., Hilgert, I., Paces, J., Simeoni, L., Kliche, S., Merten, C., Schraven, B., and Horejsí, V. (2003). LIME: a new membrane Raft-associated adaptor protein involved in CD4 and CD8 coreceptor signaling. J. Exp. Med. 198, 1453-1462.

Brdika, T., Pavlistova, D., Albrecht, L., Bruyns, E., and Schraven, B. (2000). Phosphoprotein associated with glycosphingolipid-enriched microdomains (PAG), a novel ubiquitously expressed transmembrane adaptor protein, binds the protein tyrosine kinase Csk and is involved in regulation of T cell activation. J. Exp. Med. 191, 1591-1604.

Bromann, P. A., Korkaya, H., and Courtneidge, S. A. (2004). The interplay between Src family kinases and receptor tyrosine kinases. Oncogene 23, 7957-7968.

Campi, G., Varma, R., and Dustin, M. L. (2005). Actin and agonist MHCpeptide complex-dependent $\mathrm{T}$ cell receptor microclusters as scaffolds for signaling. J. Exp. Med. 202, 10311036.

Chen, I.-J., Chen, H.-L., and Demetriou, M. (2007). Lateral compartmentalization of T cell receptor versus CD45 by galectin- $N$-glycan binding and microfilaments coordinate basal and activation signaling. J. Biol. Chem. 282, 35361-35372.

Chichili, G. R., Westmuckett, a. D., and Rodgers, W. (2010). T cell signal regulation by the actin cytoskeleton. J. Biol. Chem. 285, 14737-14746.

Davidson, D., Bakinowski, M., Thomas, M. L., Horejsi, V., and Veillette, A. (2003). Phosphorylation-dependent regulation of $\mathrm{T}$-cell activation by
PAG/Cbp, a lipid raft-associated transmembrane adaptor. Mol. Cell. Biol. 23, 2017-2028.

Davis, S. J., and van der Merwe, P. A. (2006). The kinetic-segregation model: TCR triggering and beyond. Nat. Immunol. 7, 803-809.

D'Oro, U., and Ashwell, J. D. (1999). Cutting edge: the CD45 tyrosine phosphatase is an inhibitor of Lck activity in thymocytes. J. Immunol. 162, 1879-1883.

Douglass, A. D., and Vale, R. D. (2005). Single-molecule microscopy reveals plasma membrane microdomains created by protein-protein networks that exclude or trap signaling molecules in $\mathrm{T}$ cells. Cell 121, 937-950.

Duplay, P., Thome, M., Hervé, F., and Acuto, O. (1994). p56lck interacts via its src homology 2 domain with the ZAP-70 kinase. J. Exp. Med. 179, 1163-1172.

Dustin, M. L. (2009). The cellular context of $\mathrm{T}$ cell signaling. Immunity 30 , 482-492.

Eck, M. J., Atwell, S. K., Shoelson, S. E., and Harrison, S. C. (1994). Structure of the regulatory domains of the Srcfamily tyrosine kinase Lck. Nature 368, 764-769.

Edmonds, S. D., and Ostergaard, H. L. (2002). Dynamic association of CD45 with detergent-insoluble microdomains in $\mathrm{T}$ lymphocytes. $J$. Immunol. 169, 5036-5042.

Ehrlich, L. I. R., Ebert, P. J. R., Krummel, M. F., Weiss, A., and Davis, M. M. (2002). Dynamics of p56lck translocation to the $\mathrm{T}$ cell immunological synapse following agonist and antagonist stimulation. Immunity 17 , 809-822.

Filipp, D., Zhang, J., Leung, B. L., Shaw, A., Levin, S. D., Veillette, A., and Julius, M. (2003). Regulation of Fyn through translocation of activated Lck into lipid rafts. J. Exp. Med. 197, 1221-1227.

Fragoso, R., Ren, D., Zhang, X., Su, M. W.-C., Burakoff, S. J., and Jin, Y.-J. (2003). Lipid raft distribution of CD4 depends on its palmitoylation and association with Lck, and evidence for CD4induced lipid raft aggregation as an additional mechanism to enhance CD3 signaling. J. Immunol. 170, 913-921.

Freiberg, B. A., Kupfer, H., Maslanik, W., Delli, J., Kappler, J., Zaller, D. M., and Kupfer, A. (2002). Staging and resetting $\mathrm{T}$ cell activation in SMACs. Nat. Immunol. 3, 911-917.

Furukawa, T. (1994). Specific interaction of the CD45 protein-tyrosine phosphatase with tyrosine-phosphorylated CD3ל chain. Proc. Natl. Acad. Sci. 91, 10928-10932.

Gervais, F. G., and Veillette, A. (1995) The unique amino-terminal domain of p56lck regulates interactions with tyrosine protein phosphatases in $\mathrm{T}$ lymphocytes. Mol. Cell. Biol. 15, 2393-2401.

Grakoui, A., Bromley, S. K., Sumen, C., Davis, M. M., Shaw, A. S., Allen, P. M., and Dustin, M. L. (1999). The immunological synapse: a molecular machine controlling T cell activation. Science 285, 221-227.

Granum, S., Andersen, T. C. B., Sørlie, M., Jørgensen, M., Koll, L., Berge, T., Lea, T., Fleckenstein, B., Spurkland, A., and Sundvold-Gjerstad, V. (2008). Modulation of Lck function through multisite docking to $\mathrm{T}$ cell-specific adapter protein. J. Biol. Chem. 283, 21909-21919.

Holdorf, A. D., Lee, K.-H., Burack, W. R., Allen, P. M., and Shaw, A. S. (2002). Regulation of Lck activity by CD4 and CD28 in the immunological synapse. Nat. Immunol. 3, 259-264.

Hur, E. M., Son, M., Lee, O.-H., Choi, Y. B., Park, C., Lee, H., and Yun, Y. (2003). LIME, a novel transmembrane adaptor protein, associates with p56lck and mediates T cell activation. J. Exp. Med. 198, 1463-1473.

Ike, H., Kosugi, A., Kato, A., Iino, R., Hirano, H., Fujiwara, T., Ritchie, K., and Kusumi, A. (2003). Mechanism of Lck recruitment to the T-cell receptor cluster as studied by singlemolecule-fluorescence video imaging. Chemphyschem 4, 620-626.

Johnson, K. G. (2000). A supramolecular basis for CD45 tyrosine phosphatase regulation in sustained $\mathrm{T}$ cell activation. Proc. Nat. Acad. Sci. U.S.A. 97, 10138-10143.

Jordan, S., and Rodgers, W. (2003). $\mathrm{T}$ cell glycolipid-enriched membrane domains are constitutively assembled as membrane patches that translocate to immune synapses. J. Immunol. 171, 78-87.

Kabouridis, P. S., Isenberg, D. A., and Jury, E. C. (2011). A negatively charged domain of LAT mediates its interaction with the active form of Lck. Mol. Membr. Biol. 28, 487-494.

Kaizuka, Y., Douglass, A. D., Vardhana, S., Dustin, M. L., and Vale, R. D. (2009). The coreceptor CD2 uses plasma membrane microdomains to transduce signals in $\mathrm{T}$ cells. J. Cell Biol. 185, 521-534.

Kawabuchi, M., Satomi, Y., Takao, T., Shimonishi, Y., Nada, S., Nagai, K., Tarakhovsky, A., and Okada, M.
(2000). Transmembrane phosphoprotein $\mathrm{Cbp}$ regulates the activities of Src-family tyrosine kinases. Nature 404, 999-1003.

Kim, P. W., Sun, Z.-Y. J., Blacklow, S. C., Wagner, G., and Eck, M. J. (2003). A zinc clasp structure tethers Lck to T cell coreceptors CD4 and CD8. Science 301, 1725-1728.

Lee-Fruman, K. K., Collins, T. L., and Burakoff, S. J. (1996). Role of the Lck Src homology 2 and 3 domains in protein tyrosine phosphorylation. J. Biol. Chem. 271, 25003-25010.

Leupin, O., Zaru, R., Laroche, T., Müller, S., and Valitutti, S. (2000). Exclusion of CD45 from the T-cell receptor signaling area in antigen-stimulated T lymphocytes. Curr. Biol. 10, 277-280.

Lewis, L., Chung, C., Chen, J., Parnes, J., Moran, M., Patel, V., and Miceli, M. (1997). The Lck SH2 phosphotyrosine binding site is critical for efficient TCR-induced processive tyrosine phosphorylation of the zeta-chain and IL-2 production. J. Immunol. 159, 2292-2300.

Li, Q.-J., Dinner, A. R., Qi, S., Irvine, D. J., Huppa, J. B., Davis, M. M., and Chakraborty, A. K. (2004). CD4 enhances $\mathrm{T}$ cell sensitivity to antigen by coordinating Lck accumulation at the immunological synapse. Nat. Immunol. 5, 791-799.

Lillemeier, B. F., Mörtelmaier, M. A., Forstner, M. B., Huppa, J. B., Groves, J. T., and Davis, M. M. (2010). TCR and Lat are expressed on separate protein islands on $\mathrm{T}$ cell membranes and concatenate during activation. Nat. Immunol. 11, 90-96.

Lin, R. S., Rodriguez, C., Veillette, A., and Lodish, H. (1998). Zinc is essential for binding of p56lck to CD4 and CD8alpha. J. Biol. Chem. 273, 32878-32882.

Locksley, R. M., Reiner, S. L., Hatam, F., Littman, D. R., and Killeen, N. (1993). Helper T cells without CD4: control of leishmaniasis in CD4deficient mice. Science 261, 1448 1451.

Marti, F., Garcia, G. G., Lapinski, P. E., MacGregor, J. N., and King, P. D. (2006). Essential role of the $T$ cell-specific adapter protein in the activation of LCK in peripheral T cells. J. Exp. Med. 203, 281-287.

McNeill, L., Salmond, R. J., Cooper, J. C., Carret, C. K., Cassady-Cain, R. L., Roche-Molina, M., Tandon, P., Holmes, N., and Alexander, D. R. (2007). The differential regulation of Lck kinase phosphorylation sites by CD45 is critical for T cell receptor signaling responses. Immunity 27 , 425-437. 
Monks, C. R., Freiberg, B. A., Kupfer, H., Sciaky, N., and Kupfer, A. (1998). Three-dimensional segregation of supramolecular activation clusters in T cells. Nature 395, 82-86.

Munro, S. (2003). Lipid rafts: elusive or illusive? Cell 115, 377-388.

Ng, D. H., Watts, J. D., Aebersold, R., and Johnson, P. (1996). Demonstration of a direct interaction between p56[IMAGE] and the cytoplasmic domain of CD45 in vitro. J. Biol. Chem. 271, 1295-1300.

Nichols, B. (2005). Cell biology: without a raft. Nature 436, 638-639.

Nika, K., Soldani, C., Salek, M., Paster, W., Gray, A., Etzensperger, R., Fugger, L., Polzella, P., Cerundolo, V., and Dushek, O. (2010). Constitutively active Lck kinase in $\mathrm{T}$ cells drives antigen receptor signal transduction. Immunity 32, 766-777.

Nika, K., Tautz, L., Arimura, Y., Vang, T., Williams, S., and Mustelin, T. (2007). A weak Lck tail bite is necessary for Lck function in T cell antigen receptor signaling. J. Biol. Chem. 282, 36000-36009.

Palacios, E. H., and Weiss, A. (2004). Function of the Src-family kinases, Lck and Fyn, in T-cell development and activation. Oncogene 23, 79908000 .

Parsons, S. J., and Parsons, J. T. (2004). Src family kinases, key regulators of signal transduction. Oncogene 23, 7906-7909.

Paster, W., Paar, C., Eckerstorfer, P., Jakober, A., Drbal, K., Schütz, G. J., Sonnleitner, A., and Stockinger, H. (2009). Genetically encoded Förster resonance energy transfer sensors for the conformation of the Src family kinase Lck. J. Immunol. 182, 21602167.

Pawson, T. (2004). Specificity in signal transduction: from phosphotyrosine-SH2 domain interactions to complex cellular systems. Cell 116, 191-203.

Rodgers, W., Farris, D., and Mishra, S. (2005). Merging complexes: properties of membrane raft assembly during lymphocyte signaling. Trends Immunol. 26, 97-103.

Rodgers, W., and Rose, J. K. (1996). Exclusion of CD45 inhibits activity of p56lck associated with glycolipidenriched membrane domains. J. Cell Biol. 135, 1515-1523.
Rozdzial, M. M., Pleiman, C. M., Cambier, J. C., and Finkel, T. H. (1998). pp56Lck mediates TCR zetachain binding to the microfilament cytoskeleton. J. Immunol. 161, 54915499.

Rudd, C. E., Trevillyan, J. M., Dasgupta, J. D., Wong, L. L., and Schlossman, S. F. (1988). The CD4 receptor is complexed in detergent lysates to a protein-tyrosine kinase (pp58) from human $\mathrm{T}$ lymphocytes. Proc. Natl. Acad. Sci. U.S.A. 85, 51905194.

Salmond, R. J., Filby, A., Pirinen, N., Magee, A. I., and Zamoyska, R. (2011). Mislocalization of Lck impairs thymocyte differentiation and can promote development of thymomas. Blood 117, 108-117.

Saunders, A. E., and Johnson, P. (2010). Modulation of immune cell signaling by the leukocyte common tyrosine phosphatase, CD45. Cell. Signal. 22, 339-348.

Schilham, M. W., Fung-Leung, W. P., Rahemtulla, A., Kuendig, T., Zhang, L., Potter, J., Miller, R. G., Hengartner, H., and Mak, T. W. (1993). Alloreactive cytotoxic $\mathrm{T}$ cells can develop and function in mice lacking both CD4 and CD8. Eur. J. Immunol. 23, 1299-1304.

Schoenborn, J. R., Tan, Y. X., Zhang, C., Shokat, K. M., and Weiss, A. (2011). Feedback circuits monitor and adjust basal Lck-dependent events in $\mathrm{T}$ cell receptor signaling. Sci. Signal. 4, ra59.

Secrist, J. P., Burns, L. A., Karnitz, L., Koretzky, G. A., and Abraham, R. T. (1993). Stimulatory effects of the protein tyrosine phosphatase inhibitor, pervanadate, on T-cell activation events. J. Biol. Chem. 268, 5886-5893.

Seminario, M.-C., and Bunnell, S. C. (2008). Signal initiation in Tcell receptor microclusters. Immunol. Rev. 221, 90-106.

Shenoy-Scaria, A. M., Link, D. C., and Douglas, M. L. (1994). Cysteine3 of Src family protein tyrosine kinase determines palmitoylation and localization in caveolae. J. Cell Biol. 126, 353-363.

Stefanová, I., Hemmer, B., Vergelli, M., Martin, R., Biddison, W. E., and Germain, R. N. (2003). TCR ligand discrimination is enforced by competing ERK positive and SHP1 negative feedback pathways. Nat. Immunol. 4, 248-254.

Straus, D. B., Chan, A. C., and Weiss, A. (1996). SH2 domain function is essential for the role of the Lck tyrosine kinase in $\mathrm{T}$ cell receptor signal transduction. J. Biol. Chem. 271, 9976-9981.

Tavano, R., Contento, R. L., Baranda, S. J., Soligo, M., Tuosto, L., Manes, S., and Viola, A. (2006). CD28 interaction with filamin-A controls lipid raft accumulation at the T-cell immunological synapse. Nat. Cell Biol. 8, 1270-1276.

Varma, R., Campi, G., Yokosuka, T., Saito, T., and Dustin, M. L. (2006). $\mathrm{T}$ cell receptor-proximal signals are sustained in peripheral microclusters and terminated in the central supramolecular activation cluster. Immunity 25, 117-127.

Van Laethem, F., Sarafova, S. D., Park, J.-H., Tai, X., Pobezinsky, L., Guinter, T. I., Adoro, S., Adams, A., Sharrow, S. O., Feigenbaum, L., and Singer, A. (2007). Deletion of CD4 and CD8 coreceptors permits generation of alphabetaT cells that recognize antigens independently of the MHC. Immunity 27, 735-750.

Veillette, A., Bookman, M. A., Horak, E. M., and Bolen, J. B. (1988). The CD4 and CD8 T cell surface antigens are associated with the internal membrane tyrosine-protein kinase p56lck. Cell 55, 301-308.

Wange, R. L., and Samelson, L. E. (1996). Complex complexes: signaling at the TCR. Immunity 5, 197-205. Williamson, D. J., Owen, D. M., Rossy, J., Magenau, A., Wehrmann, M., Gooding, J. J., and Gaus, K. (2011). Pre-existing clusters of the adaptor Lat do not participate in early $\mathrm{T}$ cell signaling events. Nat. Immunol. 12, 655-662.

Wong, N. K. Y., Lai, J. C. Y., Birkenhead, D., Shaw, A. S., and Johnson, P. (2008). CD45 down-regulates Lckmediated CD44 signaling and modulates actin rearrangement in T cells. $J$. Immunol. 181, 7033-7043.

Xu, H., and Littman, D. R. (1993). A kinase-independent function of Lck in potentiating antigen-specific $\mathrm{T}$ cell activation. Cell 74, 633-643.

Xu, W., Doshi, A., Lei, M., Eck, M. J., and Harrison, S. C. (1999). Crystal structures of c-Src reveal features of its autoinhibitory mechanism. Mol. Cell 3, 629-638.

Yamaguchi, H., and Hendrickson, W. A. (1996). Structural basis for activation of human lymphocyte kinase Lck upon tyrosine phosphorylation. Nature 384, 484-489.

Yamasaki, S., Takamatsu, M., and Iwashima, M. (1996). The kinase, $\mathrm{SH} 3$, and $\mathrm{SH} 2$ domains of Lck play critical roles in T-cell activation after ZAP-70 membrane localization. $\mathrm{Mol}$. Cell. Biol. 16, 7151-7160.

Zhang, H., Cordoba, S.-P., Dushek, O., and Anton van der Merwe, P. (2011). Basic residues in the $T$-cell receptor $\zeta$ cytoplasmic domain mediate membrane association and modulate signaling. Proc. Natl. Acad. Sci. U.S.A. 108, 19323-19328.

Zikherman, J., Jenne, C., Watson, S., Doan, K., Raschke, W., Goodnow, C. C., and Weiss, A. (2010). CD45-Csk phosphatase-kinase titration uncouples basal and inducible $\mathrm{T}$ cell receptor signaling during thymic development. Immunity 32, 342-354.

Conflict of Interest Statement: The authors declare that the research was conducted in the absence of any commercial or financial relationships that could be construed as a potential conflict of interest.

Received: 25 January 2012; paper pending published: 13 April 2012; accepted: 04 June 2012; published online: 19 June 2012.

Citation: Rossy J, Williamson DJ and Gaus K (2012) How does the kinase Lck phosphorylate the T cell receptor? Spatial organization as a regulatory mechanism. Front. Immun. 3:167. doi: 10.3389/ fimmu.2012.00167

This article was submitted to Frontiers in $T$ Cell Biology, a specialty of Frontiers in Immunology.

Copyright (c) 2012 Rossy, Williamson and Gaus. This is an open-access article distributed under the terms of the Creative Commons Attribution Non Commercial License, which permits noncommercial use, distribution, and reproduction in other forums, provided the original authors and source are credited. 